\title{
Glukosesensor auf Kasse: Wer zahlt am Ende drauf?
}

\author{
Seit vielen Jahren träumen Patienten und Ärzte vom \\ alltagstauglichen Glukosesensor. Nun ist es endlich \\ soweit. Der Gemeinsame Bundesausschuß (GBA) hat \\ die Kostenübernahme durch die gesetzliche Kran- \\ kenkassen unter bestimmten Voraussetzungen mög- \\ lich gemacht (Kasten). Bei der Umsetzung gibt es \\ allerdings reichlich Probleme.
}

Wie so oft existieren auch bei der Umsetzung dieser neuen Richtlinie gewaltige Unterschiede zwischen den Bundesländern. Die Darstellung berücksichtigt nur die Gegebenheiten in Bayern. Real-Time Continuous Glucose Monitoring (rtCGM-)Systeme verfügen zwingend über ein Alarmsystem. Sie sind mit dem Bescheid des GBA nun als Hilfsmittel anerkannt (die FlashGlukose-Messung ist davon ausgenommen, sie hat kein Alarmsystem). Folglich erhält der Patient ein Hilfsmittelrezept. Dieses darf nur von qualifizierten Diabetologen (Deutsche Diabetesgesellschaft (DDG) oder Bayerische Landesärztekamme (BLÄK) ausgestellt werden. Grundsätzlich ist der Patient der Leistungsempfänger und muss bei der Krankenkasse den Antrag auf Kostenübernahme abgeben. Hierzu hat die Arbeitsgemeinschaft für Diabetestechnologie (AGDT) der DDG ein Antragsformular auf ihrer Homepage bereitgestellt [1]. Die Krankenkasse hat nun innerhalb von 3 Wochen (Neufassung der Hilfsmittelverordnungsregelung $\$ 13$ SGB V Abs. 3a vom 4.4.2017) oder bei Weiterleitung an den Medizinischen Dienst der Krankenkasse (MDK) nach 5 Wochen über die Genehmigung zu entscheiden.

\section{Problemfeld Nr. 1: die Voraussetzungen}

Wird der MDK seitens der Krankenkasse eingeschaltet, muss der Patient eine Dokumentation der aktuellen Blutzucker-(BZ-)situation einreichen. Der GBA hat hierzu keine Vorgaben gemacht. Schaltet die Kasse den MDK ein, so werden derzeit von den Gutachtern sehr unterschiedliche Anforderungen gestellt. Grundsätzlich gilt: die Dokumentation von BZ-Werten genügt nicht, auch BE-Mengen, Insulindosen und besondere Ereignisse wie Sport müssen protokolliert sein. Unklar ist bislang, über welchen Zeitraum dies zu erfolgen hat. Die Fachkommission Diabetes Bayern (FKDB) ist aktuell im Gespräch mit dem MDK Bayern, um einen Konsens bezüglich der Anforderungen zu erzielen.

GBA-Bescheid Real-Time-Glukosemessung vom 7.9.2016

Die rtCGM mit Real-Time-Messgeräten ist für Diabetiker, die einer intensivierten Insulinbehandlung (ICT) bedürfen, eine Leistung der gesetzlichen Krankenkassen. Aber nur, wenn folgende Voraussetzungen erfüllt sind:

- Es sind Patienten mit ICT, die in dieser Therapie geschult sind und sie anwenden (also Typ 1, Typ 2 oder andere Diabetesformen mit ICT) und

- Patienten, die die individuellen Ziele mit den bisher verfügbaren Therapien und mit Beachtung der Lebenssituation nicht erreichen konnten.

- Und es muss das individuelle Therapieziel dokumentiert sowie die Schulung des Patienten auf ein rtCGM-System sichergestellt sein.

\section{Problemfeld Nr. 2: die Patientenschulung}

Aus diabetologischer Sicht ist ein rtCGM-System für eine große Patientengruppe definitiv zu begrüßen. Allerdings muss jeder Anwender im Umgang damit geschult werden, denn es gibt auch Risiken (z. B. zu frühe Korrekturen auf Trendpfeile und dadurch Hypoglykämien). Hierzu steht ein Schulungsprogramm zur Verfügung (Spectrum), dass von der AGDT entwickelt wurde. In 7 Modulen werden u. a. die Einstellung von Alarmgrenzen, Interpretation von Trendpfeilen und technische Fragen (Messgenauigkeit, Hautprobleme etc.) erörtert. Aber: Bisher gibt es keine adäquate extrabudgetäre Abrechnungsziffer für diese Schulung.

Seit dem 1.4.2017 wurde der Einheitliche Bewertungsmaßstab (EBM) um 3 neue Gebührenpositionen ergänzt. Die 3 Leistungen sind inhaltsgleich und können nach vorheriger Antragstellung bei der Kassenärztlichen Vereinigung und dem Nachweis entsprechender Qualifikation wie folgt abgerechnet werden:

- GOP 03355: Allgemeinmediziner, Internisten (HA) mit Zusatzbezeichnung „Diabetologi“"Qualifikation „Diabetologie DDG“ - GOP 04590 Fachärzte für Kinder- und Jugendmedizin mit Zusatzbezeichnung „Kinder-Endokrinologie u. Diabetologie“ oder "Diabetologie“ oder Qualifikation „Diabetologie DDG“

- GOP 13360 Fachärzte für Innere Medizin und Endokrinologie und Diabetologie sowie andere Internisten (FA) mit Zusatzbezeichnung „Diabetologie“ o. Qualifikation „Diabetologie DDG“ Die EBM-Bewertung beträgt 72 Punkte (aktuell ca 7,58 €) und kann bei mindestens 10 -minütiger Anleitung im Umgang mit einem rtCGM-System 10mal im Krankheitsfall berechnet werden. Aber: Diese neue EBM-Ziffer bildet nicht den Aufwand einer qualifizierten Schulung ab (aktuell nur mit „Spectrum “ möglich). Für eine „Schulungsstunde“ sind ca. 90 Minuten nötig.

Daher frage ich: Wie kann der Gesetzgeber eine Schulung als Grundvoraussetzung für die Kostenerstattung fordern, diese aber nicht adäquat honorieren? Den bayrischen Berufsverbänden wurde definitiv kein Mitspracherecht eingeräumt - wie sieht es damit in anderen Bundesländern aus? Ist unsere Leistung so unwesentlich, dass sie nicht mehr als $75 €$ (für 7 Schulungseinheiten á etwa 90 Minuten) wert ist?

- Hier wäre es sehr hilfreich, Rückmeldungen von diabetologisch tätigen Kollegen aus anderen Bundesländern zu erhalten, gern mitthilfe einer E-Mail an mich.

1. Link: http://www.diabetes-technologie.de/images/pdfs/ glukosemonitoring-downloads/rtCGM-Kostenerstattung-version0-6-LH-2016-10-19-DDG.pdf.

Dr. med. Veronika Hollenrieder

FÄ für Innere Medizin, Diabetologin DDG, Vorstandsmitglied FKDB Hauptstr. 14, 82008 Unterhaching

veronika@praxis-hollenrieder.de 